CERN-TH-6460/92

\title{
Smooth Bosonization: The Cheshire Cat Revisited
}

\author{
P.H. DAMGAARD \\ CERN - Geneva \\ H.B. NiElsen and R. Sollacher \\ The Niels Bohr Institute \\ Blegdamsvej 17 \\ DK-2100 Copenhagen, Denmark
}

\begin{abstract}
A general method for deriving effective Lagrangians is used to establish a continuous bosonization of a fermionic theory. This is achieved by introducing new fields in the path integral, and choosing different gauges in an equivalent version of the theory containing also a bosonic field. We illustrate this idea by showing the equivalence of fermions and bosons in $(1+1)$ dimensions. We also demonstrate a smooth transition in space from a bosonic to a fermionic representation, providing a new class of soft Cheshire Cat bag models, as well as a smooth transition in momentum space introducing effective low and high energy fields.
\end{abstract}

CERN-TH-6460/92

April 1992 



\section{Introduction}

The advantage of having different field representations of the same theory can be illustrated by an example in $(1+1)$ dimensions. The quantum theory of the massive Thirring model

$$
\mathcal{L}_{M T}=\bar{\psi} i \not \partial \psi-m \bar{\psi} \psi-\frac{g}{2} \bar{\psi} \gamma^{\mu} \psi \bar{\psi} \gamma_{\mu} \psi
$$

is known to be equivalent to the quantized Sine-Gordon model $[1,2,3]$ :

$$
\mathcal{L}_{S G}=\frac{1}{2} \partial^{\mu} \theta \partial_{\mu} \theta+\frac{\alpha_{0}}{\beta^{2}}(\cos \beta \theta-1)
$$

This equivalence is conveniently expressed in terms of the well-known abelian bosonization rules:

$$
\begin{aligned}
\bar{\psi} \gamma^{\mu} \psi & =-\frac{\beta}{2 \pi} \epsilon^{\mu \nu} \partial_{\nu} \theta \\
Z m \bar{\psi} \frac{1 \pm \gamma_{5}}{2} \psi & =-\frac{\alpha_{0}}{\beta^{2}} e^{\mp i \beta \theta}
\end{aligned}
$$

Here, $Z$ is a constant depending on the regularization of both representations. In addition, there is a relation between the coupling constants:

$$
1+\frac{g}{\pi}=\frac{4 \pi}{\beta^{2}}
$$

Throughout this paper we use the conventions (see [1]):

$$
\gamma_{5}=\gamma_{0} \gamma_{1} \quad, \quad g_{\mu \nu}=\operatorname{diag}(1,-1) \quad, \quad \epsilon_{01}=-\epsilon_{10}=1 .
$$

The definition of $\gamma_{5}$ and the algebra of $\gamma$-matrices in $(1+1)$ dimensions implies the equation

$$
\gamma_{\mu} \gamma_{5}=-\epsilon_{\mu \nu} \gamma^{\nu}
$$

showing that the axial current and the vector current are not independent. As a consequence, one has automatically one more bosonization relation:

$$
\bar{\psi} \gamma^{\mu} \gamma_{5} \psi=\frac{\beta}{2 \pi} \partial^{\mu} \theta
$$

The two different field representations (1) and (2) describing the same physics are useful in different regimes. If the coupling constant $g$ is small, then the massive Thirring model is obviously more convenient; the self interaction can be treated perturbatively (except perhaps for high density). According to (4), these values of $g$ correspond to $\beta \simeq$ $2 \sqrt{\pi}$ which cannot be regarded as small. Nevertheless, we have a system of weakly self interacting pointlike fermions. If, in contrast, $g$ is large then the fermions are surrounded by a highly polarized Dirac sea. The fermions are now extended objects which are best described by the Sine-Gordon model; $g$ being large implies small $\beta$ which allows for a semiclassical approximation. For topological charge one, this yields solitons with a size of the order $\sqrt{\alpha_{0}}$.

Another example illuminating the need for a proper quasi particle picture is given by the concept of a hybrid bag. This idea arose out of the observation that at small distances the baryons appear as a bound state of three pointlike quarks, whereas at large distances they are better represented as a collective state of mesons. The hybrid 
bag accounts for this fact by describing baryons as quarks confined in a bag which is surrounded by a topologically nontrivial meson field. The more refined these models were in $(3+1)$ dimensions, the less they depended on the bag radius. The extreme extrapolation of this behaviour leads to the "Cheshire Cat" principle [4], which states that physical quantities should be independent of the bag radius.

We hope that these examples convincingly illustrate the merit of different field representations of a physical system. In the $(1+1)$-dimensional example, the different representations are fully equivalent at the quantum level. This holds at both weak and strong coupling as well as at large and small length scales. Nevertheless, for calculational purposes it may be convenient to use the language of one representation in certain regimes, and vice versa. In a sense, the two descriptions are dual to each other, describing the same physics in a complementary manner.

The fact that very different choices of variables may be used to describe the same physical situation is reminiscent of the choice of gauge in, say, Yang-Mills theory. In this case different gauge choices, while ultimately yielding the same physical gauge invariant results, may be convenient in different circumstances. A question that comes to mind is whether a change of field representation can similarly be viewed as moving from one gauge slice to another, this time in a gauge invariant theory described by an enlarged set of field variables. This is indeed the case [5]. The redundancy implied by an overcomplete basis of field variables in the enlarged theory manifests itself in a new local symmetry. This local symmetry is a gauge symmetry in the conventional sense: In the Hamiltonian formulation it corresponds to a set of 1st class constraints (one for each added degree of freedom) [6]. Explicitly, suppose we perform a field enlarging change of variables

$$
\left\{\theta_{i}\right\} \rightarrow\left\{\theta_{i}, a_{\alpha}\right\}
$$

where the $a_{\alpha}(x)$ are arbitrarily chosen fields. The transformed theory is now subject to a corresponding set of constraints $\Phi_{\alpha}$ with Poisson brackets

$$
\left\{H, \Phi_{\alpha}\right\}=0 ;\left\{\Phi_{\alpha}, \Phi_{\beta}\right\}=0
$$

with $H$ being the transformed Hamiltonian. These constraints generate a local abelian gauge symmetry in the usual manner.

One example suggested in ref. [5] was that perhaps even field representations switching between bosons and fermions (as in $(1+1)$ dimensions) could be viewed as different gauge choices of a larger gauge symmetric theory in which both bosons and fermions appear on equal terms. Earlier work on path integral bosonization $[7,8,9]$ already suggested that the relevant field transformation might involve local chiral rotations of the fermions. If this point of view is tenable, it would imply that the standard bosonization and fermionization prescriptions just yield two extreme field representations of a "larger" theory which in a completely smooth way can be brought to almost any desired form inbetween. The equivalence between certain specific bosonic and fermionic theories (as exemplified above by the Massive Thirring and Sine-Gordon models) should then be far bigger, extending to a continuum of theories in which fermions and bosons appear interactively to a greater or lesser extent. We call this idea smooth bosonization.

In this paper, we shall attempt to substantiate the above program, and also present an explicit bag model construction which we think is of interest. Although our framework throughout shall be that of $(1+1)$-dimensional field theory, we will always have more 
physical settings in mind. In particular, we shall use this new tool of local bosonization gauge symmetry to reinvestigate the Cheshire Cat hypothesis of hybrid bag models. In $(3+1)$ dimensions we may never be able to construct an exact Cheshire Cat Model (CCM) describing QCD bound states and their interactions, but even an approximate CCM is presumably a qualitatively viable description of hadronic phenomenology. The $(1+1)$ dimensional theoretical laboratory can then serve as a useful guideline: On account of bosonization, here one can construct exact CCMs [4]. These models are usually realized with free massless fermions inside the bag, free massless bosons outside the bag, and an interaction between these fields only on the bag surface. This surface interaction term is uniquely determined by the usual symmetries and the Cheshire Cat criterion. The construction with two equivalent theories inside and outside the bag, together with this special surface interaction guarantees undisturbed propagation of physical information across the bag wall [4]. As such one can move the bag and change its size without changing the physical content of the theory. This allows for a continuous change from the purely fermionic representation to the purely bosonic representation by shrinking the bag radius from infinity to zero. With the new local gauge symmetry at hand, we can achieve this very directly by switching between bosonic and fermionic descriptions in different regions of space-time. This can be done even in a completely smooth manner, giving rise to $(1+1)$ dimensional "soft bag" models with a smooth and continuous transition between the two field representations. One could call these Lagrangians smooth Cheshire Cat Models. But before reaching this point, let us set up the general formalism describing smooth bosonization.

\section{The introduction of a collective field $\theta(x)$}

The discussion of the previous section has already suggested an interpretation of the soliton as a collective fermion state. How should we view this collective field variable? If we return to the bosonization prescription (3), it is well known that the composite fermion bilinears require a more careful definition on account of the short-distance singularity of the field operators. Point-splitting can serve as sufficient regularization. For example, let us define the current $j_{\mu}(x)$ on the 1.h.s. of eq. $(3)$ as $\left.\bar{\psi}(x+\epsilon) \gamma_{\mu} \psi(x)\right|_{\epsilon \rightarrow 0}$. Under a local chiral transformation,

$$
\begin{aligned}
& \bar{\psi}(x) \quad \rightarrow \quad \bar{\psi}(x) e^{i \alpha(x) \gamma_{5}} \\
& \psi(x) \rightarrow e^{i \alpha(x) \gamma_{5}} \psi(x),
\end{aligned}
$$

this regularized current transforms non-trivially:

$$
\left.\left.\bar{\psi}(x+\epsilon) \gamma_{\mu} \psi(x)\right|_{\epsilon \rightarrow 0} \rightarrow \bar{\psi}(x+\epsilon) \gamma_{\mu} \psi(x)\right|_{\epsilon \rightarrow 0}+\frac{1}{\pi} \epsilon_{\mu \nu} \partial^{\nu} \alpha(x)
$$

implying, according to eq. (3), that a chiral transformation on $\bar{\psi}$ and $\psi$ is equivalent to a shift on the Sine-Gordon field $\theta: \theta(x) \rightarrow \theta(x)-\frac{2}{\beta} \alpha(x)$. This in turn indicates that the soliton field $\theta(x)$ describes the dynamics of the chiral phase of $\psi(x)$, which seems to be the only physical one in $(1+1)$ dimensions - at least for models of this type. The obvious method to probe this degree of freedom is to perform a chiral transformation of the fermion fields. In the functional integral, such transformations are accompagnied by a Jacobian factor, part of which is the well known chiral anomaly. It has been recognized for some time that this Jacobian plays an important role for bosonization in the functional integral approach [7]. 
To begin, we first restrict ourselves to the case of massless fermions coupled to external vector and axial vector fields. The fermions, of the Dirac kind, are described by complex two-component spinors. A matrix basis for the $\gamma$-matrix algebra is given by $\left\{1, \gamma_{0}, \gamma_{1}, \gamma_{5}\right\}$, all other matrices being linear combinations; we use the conventions of eq.(5). The generating functional is given by

$$
\begin{aligned}
\mathcal{Z}[V, A] & =\int \mathcal{D}[\psi, \bar{\psi}] e^{i \int d^{2} x \mathcal{L}} \\
\mathcal{L} & =\bar{\psi}\left(i \not \partial+V+\not \mathcal{A} \gamma_{5}\right) \psi
\end{aligned}
$$

In addition, we assume a regularization which preserves vector current conservation. This is easily achieved by, e.g., a Pauli-Villars regularization [10]. The restriction in eq. (12) to fermions coupling only to vector and axial vector sources, $V_{\mu}$ and $A_{\mu}$, is not as trivial as may appear at first sight. For example, if we add a term $\sim V_{\mu} V^{\mu}$ to the Lagrangian, and integrate over the source $V_{\mu}$ (now really an auxiliary field), we recover the massless Thirring model. To make the generating functional (12) complete in the sense that it can represent the full Massive Thirring model of eq. (1), we only need to add sources for the scalar and pseudoscalar densities $\bar{\psi} \psi$ and $\bar{\psi} \gamma_{5} \psi$ as well. This will be considered in section 5.

As already pointed out, the collective degree of freedom can be probed by a local chiral transformation:

$$
\psi(x)=e^{i \theta(x) \gamma_{5}} \chi(x) \quad, \quad \bar{\psi}(x)=\bar{\chi}(x) e^{i \theta(x) \gamma_{5}} .
$$

This change of integration variables does not affect the generating functional, but changes the Lagrangian:

$$
\begin{aligned}
\mathcal{Z}[V, A]= & \int \mathcal{D}[\chi, \bar{\chi}] e^{i \int d^{2} x \mathcal{L}^{\prime}(\bar{\chi}, \chi, \theta)} \\
\mathcal{L}^{\prime}(\bar{\chi}, \chi, \theta)= & \bar{\chi}\left(i \not \partial+V+\not \gamma_{5}-\not \partial \theta \gamma_{5}\right) \chi \\
& +\frac{1}{2 \pi} \partial_{\mu} \theta \partial^{\mu} \theta+V_{\mu} \frac{1}{\pi} \epsilon^{\mu \nu} \partial_{\nu} \theta-A_{\mu} \frac{1}{\pi} \partial^{\mu} \theta
\end{aligned}
$$

The last line is the contribution of the Jacobian of this chiral transformation. Since we have just performed a change of variables in the path integral, we can integrate over $\theta$ without changing the generating functional. The field $\theta(x)$ has then become dynamical.

As previously advertised, our enlarged Lagrangian now exhibits a local gauge symmetry. The symmetry transformation is

$$
\chi(x) \rightarrow e^{i \alpha(x) \gamma_{5}} \chi(x) \quad, \quad \bar{\chi}(x) \rightarrow \bar{\chi}(x) e^{i \alpha(x) \gamma_{5}} \quad, \quad \theta(x) \rightarrow \theta(x)-\alpha(x)
$$

It is important to include the chiral Jacobian of the fermionic measure in order to recognize this as a symmetry transformation.

Gauge fixing in the standard Faddeev-Popov fashion, the generating functional (12) then reads

$$
\begin{aligned}
\mathcal{Z}[V, A]= & \int \mathcal{D}[\chi, \bar{\chi}] \mathcal{D}[\theta] \delta(\Phi[\theta])\left|\operatorname{det}\left(\frac{\delta \Phi}{\delta \theta}\right)\right| e^{i \int d^{2} x \mathcal{L}^{\prime}} \\
\mathcal{L}^{\prime}= & \bar{\chi}\left(i \not \partial+V+\not \gamma_{5}-\not \partial \theta \gamma_{5}\right) \chi \\
& +\frac{1}{2 \pi} \partial_{\mu} \theta \partial^{\mu} \theta+V_{\mu} \frac{1}{\pi} \epsilon^{\mu \nu} \partial_{\nu} \theta-A_{\mu} \frac{1}{\pi} \partial^{\mu} \theta
\end{aligned}
$$


It is easy to check that the trivial gauge $\Phi[\theta]=\theta$ corresponds to the original representation (12).

Consider now, for simplicity, the case where $A_{\mu}=0$ (no axial vector coupling). The new field $\theta(x)$ couples to the vector source $V_{\mu}$ as $(1 / 2 \pi) \theta(x) \epsilon_{\mu \nu} F^{\mu \nu}(x)$, where $F_{\mu \nu}=$ $\partial_{\mu} V_{\nu}-\partial_{\nu} V_{\mu}$ is the abelian field strength. So just as in $(3+1)$ dimensions, where the field $\theta(x)$ couples to the four-dimensional Pontryagin density $F_{\mu \nu} \tilde{F}^{\mu \nu}[5]$, also here in $(1+1)$ dimensions $\theta(x)$ couples to the topological term (now $\epsilon_{\mu \nu} F^{\mu \nu}(x)$ ). Indeed, we see from eq. (16) that $\theta(x)$ is just like an axion field, except that it has no tree-level self-interactions.

Inn the usual manner, we can represent the gauge fixing $\delta$-functional by its functional Fourier transform, and the Faddeev-Popov determinant as a functional integral over fermionic ghost fields $\bar{c}(x), c(x)$ :

$$
\begin{aligned}
\mathcal{Z}[V, A]= & \int \mathcal{D}[\chi, \bar{\chi}] \mathcal{D}[\theta] \mathcal{D}[b, \bar{c}, c] e^{i \int d^{2} x \mathcal{L}_{B R S T}} \\
\mathcal{L}_{B R S T}= & \bar{\chi}\left(i \not \partial+V+A \gamma_{5}-\not \partial \theta \gamma_{5}\right) \chi \\
& +\frac{1}{2 \pi} \partial_{\mu} \theta \partial^{\mu} \theta+V_{\mu} \frac{1}{\pi} \epsilon^{\mu \nu} \partial_{\nu} \theta-A_{\mu} \frac{1}{\pi} \partial^{\mu} \theta \\
& +b \Phi[\theta]+\bar{c}\left(\frac{\delta \Phi}{\delta \theta}\right) c
\end{aligned}
$$

The gauge fixing term breaks the local gauge symmetry leaving us with only a global BRST symmetry

$$
\begin{aligned}
\delta_{B R S T} \bar{\chi}(x) & =i \bar{\chi}(x) \gamma_{5} c(x) \\
\delta_{B R S T} \chi(x) & =-i c(x) \gamma_{5} \chi(x) \\
\delta_{B R S T} \theta(x) & =c(x) \\
\delta_{B R S T} c(x) & =0 \\
\delta_{B R S T} \bar{c}(x) & =-b(x) \\
\delta_{B R S T} b(x) & =0,
\end{aligned}
$$

described in terms of the standard BRST multiplet of fields. It is easy to see that this set of transformations satisfies $\delta_{B R S T}^{2}=0$. We shall return to BRST quantization of the theory in the next section.

The above BRST symmetry is realized only if one carefully includes the fermionic Jacobian. In four dimensions, it is particularly easy to see that one unbroken Ward Identity of this BRST invariance equals the broken Ward Identity of chiral symmetry [5]. This holds, as it should, even in the "trivial" gauge $\Phi[\theta]=\theta=0$ mentioned above.

\section{Bosonization}

\subsection{An ANOMALOUS GaUgE FIXING CONDITION}

Let us now consider in some detail two specific gauge fixing functions which will allow us to go to purely fermionic or bosonic descriptions in a smooth manner. For this purpose let us concentrate on the axial current $J_{5}^{\mu}$ coupled to $A_{\mu}$ in (16). Recall that this current represents the original axial current $\bar{\psi} \gamma^{\mu} \gamma_{5} \psi$ as expressed in (12). The divergence of this current can be derived from e.g. the equation of motion for $\theta(x)$ :

$$
\partial_{\mu} J_{5}^{\mu}=\partial_{\mu}\left(\bar{\chi} \gamma^{\mu} \gamma_{5} \chi-\frac{1}{\pi} \partial^{\mu} \theta\right)=-\frac{1}{\pi} \partial_{\mu} A^{\mu}-\frac{1}{\pi} \epsilon^{\mu \nu} \partial_{\mu} V_{\nu}+\text { g.f. terms }
$$

Apart from the usual anomaly there is also a contribution from the gauge fixing terms in eq.(19). 
Our aim is to relate fermionic and bosonic quantities through specific gauge fixing functions. There is, of course, a huge variety of possible gauges, all being unobservable for physical processes. We use the composition of the axial current $J_{5}^{\mu}$ in (19) as a guide to find a suitable gauge. To remove the one additional field degree of freedom described by the pseudoscalar field $\theta(x)$, we take as a gauge the condition that the bosonic part $-\frac{1}{\pi} \partial^{\mu} \theta$ represents a fraction $\Delta$ of the physical current $J_{5}^{\mu}$. We can only fix one degree of freedom; therefore, we formulate the above condition in terms of the divergence of these currents:

$$
\begin{aligned}
\Phi[\theta, \bar{\chi}, \chi] & =-\partial_{\mu}\left(-\frac{1}{\pi} \partial^{\mu} \theta(x)-\Delta J_{5}^{\mu}(x)\right) \\
& =\partial_{\mu}\left(\Delta \bar{\chi}(x) \gamma^{\mu} \gamma_{5} \chi(x)+(1-\Delta) \frac{1}{\pi} \partial^{\mu} \theta(x)\right)=0
\end{aligned}
$$

In the last line we have used the definition of $J_{5}^{\mu}$ in (19). As a result, $\Phi[\ldots]$ depends not only on $\theta(x)$, but also on the fermion fields.

This has consequences for the Faddeev-Popov determinant due to the nontrivial behaviour of the fermionic axial current under a local chiral transformation. This determinant, in general, depends on the response of the gauge constraint $\Phi[. .$.$] to an infinitesimal$ gauge transformation (15). As long as $\Phi[\ldots]$ depends only on $\theta(x)$, this response is given by a derivative with respect to $\theta(x)$. Now, instead, we get:

$$
\left|\operatorname{det}\left(\frac{\delta \Phi}{\delta \alpha}\right)\right|=\left|\operatorname{det}_{(0)}\left(-\frac{1}{\pi} \partial^{2}\right)\right| .
$$

Actually, the gauge (20) is not yet complete; it defines $\theta(x)$ as a function of the fermionic axial current up to a zero mass mode $\theta_{0}(x)$, with $\partial^{2} \theta_{0}(x)=0$. We therefore have to restrict ourselves to the subspace of nonzero-modes, which is indicated by the index $(0)$ in $(21)$. To complete the gauge fixing procedure, we also have to specify a particular $\theta_{0}(x)$.

This Faddeev-Popov determinant (21) can be obtained by writing the gauge fixing factor as in (17) representing an additional interaction term $\mathcal{L}_{g . f}^{\prime}$ :

$$
\begin{aligned}
\delta(\Phi[\theta, \bar{\chi}, \chi]) & =\delta\left(\partial_{\mu} \Delta \bar{\chi} \gamma^{\mu} \gamma_{5} \chi+\frac{1}{\pi} \partial_{\mu}(1-\Delta) \partial^{\mu} \theta\right) \\
& =\int \mathcal{D}[b] e^{i \int d^{2} x b \partial_{\mu}\left(\Delta \bar{\chi} \gamma^{\mu} \gamma_{5} \chi+\frac{1}{\pi}(1-\Delta) \partial^{\mu} \theta\right)} \\
& =\int \mathcal{D}[b] e^{i \int d^{2} x \mathcal{L}_{g . f}^{\prime}} .
\end{aligned}
$$

We now have to calculate the response of the gauge fixing term $\Phi[\ldots]$ to a gauge transformation (15) for infinitesimal $\alpha(x)$. We get a contribution from the shift of $\theta(x)$ and an additional non-trivial term from the Jacobian due to the derivative coupling of $b$ to the fermion axial current, as discussed in section 2.

All these additional terms can be written as a change of the gauge fixing part $\mathcal{L}_{g . f}^{\prime}$. of the Lagrangian:

$$
\delta_{\alpha} \mathcal{L}_{g . f .}^{\prime}=-\frac{1}{\pi} b \partial_{\mu}((1-\Delta)+\Delta) \partial^{\mu} \alpha=-\frac{1}{\pi} b \partial^{2} \alpha=b \delta_{\alpha} \Phi[\ldots]
$$

From this expression, the result quoted in eq.(21) above follows ${ }^{1)}$.

1) This result is also true for the case of a space-time dependent $\Delta(x)$, which we consider later. 
The appearance of the extra, in fact anomalous, part of the Faddeev-Popov determinant can also be understood in a more algebraic manner by means of BRST quantization of the theory. We have already introduced in eq.(18) the required BRST multiplet of fields, and the associated set of transformations.

To fix the gauge in a BRST invariant way to the condition $\Phi[\theta, \bar{\chi}, \chi]=0$ of eq. (20), we subtract a term from the Lagrangian of the form $\mathcal{L}_{g f}=\delta_{B R S T}[\bar{c} \Phi]$. Since $\delta_{B R S T}^{2}=0$, such a term is by construction BRST invariant, and it precisely breaks the gauge symmetry by restricting the functional integral to the surface $\Phi[\theta, \bar{\chi}, \chi]=0$. Classically, we then get

$$
\delta_{B R S T}[\bar{c} \Phi]=-b \Phi[\theta, \bar{\chi}, \chi]-\frac{1}{\pi} \bar{c} \partial_{\mu}(1-\Delta) \partial^{\mu} c,
$$

which should be subtracted from the original Lagrangian. Here, we have made use of the grading of $\delta_{B R S T}$ and the anticommutativity of the Grassmann variables to show that $\delta_{B R S T}\left[\partial_{\mu} \bar{\chi} \gamma^{\mu} \gamma_{5} \chi\right]=0$. (Indeed, the chiral current $\bar{\chi} \gamma_{\mu} \gamma_{5} \chi$ appears naively to be a singlet under chiral transformations, and hence should have no chiral BRST variation). But we know that care in the definition of $\delta_{B R S T}\left[\bar{\chi}(x) \gamma_{\mu} \gamma_{5} \chi(x)\right]$ is required here. Thus, from eq.(11) it follows that a proper definition (the one consistent with vector current conservation) of the BRST variation of this composite operator will yield (by, e.g., point splitting, or otherwise):

$$
\left.\delta_{B R S T}\left[\bar{\chi} \gamma_{\mu} \gamma_{5} \chi\right]\right|_{r e g}=-\frac{1}{\pi} \partial_{\mu} c .
$$

The full ghost term added to the action is thus $-\frac{1}{\pi} \bar{c} \partial_{\mu}((1-\Delta)+\Delta) \partial^{\mu} c=-\frac{1}{\pi} \bar{c} \partial^{2} c$. These ghosts are therefore completely decoupled in this gauge, and when integrated out precisely give the determinant quoted above. Note that we are dealing here with a (BRST) gauge fixing situation where the gauge fixing function transforms anomalously under the given gauge (or BRST) transformation. In fact, the special case $\Delta=1$ corresponds to a gauge fixing function which classically is completely gauge invariant! Only when the anomaly is taken into account does it produce a proper gauge fixing.

\subsection{INCLUDING THE ZERO MODES}

An alternative choice of gauge relating $\bar{\chi}(x)$ and $\chi(x)$ directly to $\theta(x)$ should avoid the problems with zero modes. Formally, this can be achieved by solving (20) with respect to $\theta(x)$. This involves the inversion of $\partial^{2}$, which gives an unpleasant nonlocal term.

Instead, we now define a gauge fixing condition that can be viewed as a formal integration of $(20)$, in this way avoiding the problem of zero modes:

$$
\Phi^{\prime}[\theta, \chi, \bar{\chi}]=\Delta \int_{x_{0}}^{x} d \xi^{\nu} \bar{\chi}(\xi) \gamma_{\nu} \gamma_{5} \chi(\xi)+(1-\Delta) \frac{1}{\pi} \theta(x)=0 .
$$

The integral is a line integral connecting the points $x_{0}$ and $x$. On-shell the result is independent of the path due to vector current conservation. Different choices of $x_{0}$ then change $\Phi^{\prime}$ by a constant. However, off-shell this object is in general path-dependent. To begin, let us therefore form a fixed "fibration" of space-time, associating to each spacetime point $x$ a unique path to $x_{0}$. Since the result is path dependent off-shell, this at first sight fixes $\theta(x)$ to be dependent on the fibration. But as we shall see below, the path-dependence actually drops out once we perform the functional integrals.

The Faddeev-Popov determinant corresponding to eq. (26) is

$$
\operatorname{det}\left(\frac{\delta \Phi^{\prime}}{\delta \alpha}\right)=\operatorname{det}\left(-\frac{1}{\pi}\right),
$$


which now includes the zero mode subspace as well. The cancellation of the $\Delta$ terms comes from the anomalous shift of the axial current under a chiral transformation, analogous to the derivation of $(21)^{2)}$. Both gauges, $\Phi[\ldots]$ and $\Phi^{\prime}[\theta, \ldots]$, are equivalent for constant $\Delta$, and modulo zero modes. Although one can presumably carry the following analysis through for the gauge fixing function $\Phi[\theta, \bar{\chi}, \chi]$, the latter one (26) is more convenient for our purposes. (It shifts the problem of zero modes of $\theta$ into the question of pathdependence of the gauge fixing condition (26), an issue we shall resolve shortly).

\subsection{BOSONIZATION}

Let us first consider the particular case $\Delta=0$. The gauge $\Phi^{\prime}[\theta, \ldots]$ in $(26)$ then simply states $\theta(x)=0$. Carrying out the $\theta$-integration with this constraint immediately yields the original version (12). In detail, we have

$$
\begin{aligned}
\mathcal{Z}[V, A] & =\int \mathcal{D}[\chi, \bar{\chi}] \mathcal{D}[\theta] \delta\left(\frac{1}{\pi} \theta\right)\left|\operatorname{det}\left(-\frac{1}{\pi}\right)\right| e^{i \int d^{2} x \mathcal{L}^{\prime}} \\
& =\int \mathcal{D}[\chi, \bar{\chi}] e^{i \int d^{2} x \mathcal{L}} \\
\mathcal{L} & =\bar{\chi}\left(i \not \partial+V+A \gamma_{5}\right) \chi
\end{aligned}
$$

This is indeed the functional $\mathcal{Z}[V, A]$ in its original representation.

The more interesting case is of course $\Delta \neq 0$. For $\Delta=1$, we shall now show that we can remove all occurrences of fermionic currents, i.e., in the present case all fermionic couplings. The generating functional (16) in this case reads

$$
\begin{aligned}
\mathcal{Z}[V, A]= & \int \mathcal{D}[\chi, \bar{\chi}] \mathcal{D}[\theta] \delta\left(\int_{x_{0}}^{x} d \xi_{\mu} \bar{\chi} \gamma^{\mu} \gamma_{5} \chi\right)\left|\operatorname{det}\left(-\frac{1}{\pi}\right)\right| e^{i \int d^{2} x \mathcal{L}^{\prime \prime}} \\
\mathcal{L}^{\prime \prime}= & \bar{\chi} i \not \partial \chi+\frac{1}{2 \pi} \partial_{\mu} \theta \partial^{\mu} \theta+V_{\mu}\left(\bar{\chi} \gamma^{\mu} \chi+\frac{1}{\pi} \epsilon^{\mu \nu} \partial_{\nu} \theta\right) \\
& +A_{\mu}\left(\bar{\chi} \gamma^{\mu} \gamma^{5} \chi-\frac{1}{\pi} \partial^{\mu} \theta\right)
\end{aligned}
$$

This whole expression still looks formally dependent on the fibration of paths chosen; however, this is not the case. To see this, let us consider the change in the generating functional under an infinitesimal change of paths. It is convenient to use an integral representation of the delta function giving the gauge fixing constraint. This yields the following:

$$
\begin{aligned}
\delta_{\text {Path }} \mathcal{Z}[V, A]= & \int \mathcal{D}[\theta] \mathcal{D}[\chi, \bar{\chi}] \mathcal{D}[b]\left|\operatorname{det}\left(-\frac{1}{\pi}\right)\right| i \int d^{2} x b(x) \int_{\delta P} d^{2} \xi \partial_{\mu} \bar{\chi} \gamma^{\mu} \chi \\
& \exp \left[i \int d^{2} x \mathcal{L}^{\prime \prime}+i \int d^{2} x b(x) \int_{x_{0}}^{x} d \xi_{\mu} \bar{\chi} \gamma^{\mu} \gamma_{5} \chi\right]
\end{aligned}
$$

where the surface integral runs over the area $\delta P$ enclosed by the two nearby paths.

We now show that although we cannot use on-shell equations of motion inside the path integral, we nevertheless keep current conservation in terms of averages. To see this, we need three facts. First, the fermionic measure is invariant under local phase rotations,

$$
\bar{\chi}(x) \rightarrow e^{-i \varphi(x)} \bar{\chi}(x), \quad \chi(x) \rightarrow e^{i \varphi(x)} \chi(x)
$$

2) A chiral transformation just shifts the axial current by the gradient of the chiral angle divided by $\pi$ - which is all one needs. 
(this is automatically achieved by the regulator we are using for the fermionic measure). Secondly, both sources $V_{\mu}$ and $A_{\mu}$ couple to fermionic bilinears that are also invariant under these local phase rotations. Finally, such rotations also leave the gauge fixing function invariant. The transformation (31) then implies the following identity involving the full generating functional (29):

$$
\begin{aligned}
0 & =\frac{\delta}{\delta \varphi(x)} \ln \mathcal{Z}[V, A] \\
& =\left\langle\partial_{\mu} \bar{\chi} \gamma^{\mu} \chi\right\rangle_{V, A} .
\end{aligned}
$$

That is, the vector current is always conserved in terms of expectation values, even in the presence of the external sources $V_{\mu}$ and $A_{\mu}$. Note that this hinges crucially on these external sources coupling only to terms invariant under local phase rotations. (If they were to transform non-trivially under local rotations, while still being globally invariant, we would still find a current that would be conserved both classically and at the level of expectation values. It would, however, not coincide with $\bar{\chi} \gamma^{\mu} \chi$.). It follows straightforwardly that a stronger statement holds as well: All expectation values involving $\partial^{\mu} \bar{\chi} \gamma_{\mu} \chi$ and any object $G\left[x_{1}, \ldots, x_{n}\right]$ composed out of the fields $\theta(x), \bar{\chi}(x)$ and $\chi(x)$ at positions $x_{1}, \ldots, x_{n}$ also vanishes,

$$
\left\langle\partial^{\mu} \bar{\chi} \gamma_{\mu} \chi G\left[x_{1}, \ldots, x_{n}\right]\right\rangle_{V, A}=0
$$

whenever $G\left[x_{1}, \ldots, x_{n}\right]$ is invariant under local phase rotations.

The result of eq. (33) immediately implies

$$
\delta_{\text {Path }} \mathcal{Z}[V, A]=0,
$$

under the infinitesimal change of paths indicated by (30). So, although the gauge fixing function $\Phi^{\prime}[\theta, \bar{\chi}, \chi]$ is only independent of the chosen path on-shell, the off-shell path dependence cancels out once the gauge fixing function is taken inside the fermionic functional integral. (Any finite change of path within the same homology class can always be built up from a sequence of infinitesimal changes. We are in any case mainly concerned with a space-time of trivial topology).

Now, because of the $\delta$-function constraint in (29) we have

$$
\frac{\partial}{\partial x^{\nu}}\left\langle\int_{x_{0}}^{x} d \xi^{\mu} \bar{\chi} \gamma_{\mu} \gamma^{5} \chi\right\rangle_{V, A}=0
$$

Again, since the integral inside the expectation value will be independent of the path, this implies

$$
\left\langle\bar{\chi} \gamma^{\mu} \gamma^{5} \chi\right\rangle_{V, A}=0
$$

in the arbitrary background of sources $V_{\mu}$ and $A_{\mu}$. This trivially implies $\left\langle\bar{\chi} \gamma^{\mu} \chi\right\rangle_{V, A}=0$ as well. In fact, it is clear then that we can bring arbitrary factors of $\bar{\chi} \gamma^{\mu} \gamma^{5} \chi$ and $\bar{\chi} \gamma^{\mu} \chi$ inside the path integral, and still obtain vanishing correlators. Effectively we can therefore discard any coupling between $V_{\mu}$ and $A_{\mu}$ and these fermionic currents. This leaves us with

$$
\begin{aligned}
\mathcal{Z}[V, A] & =\int \mathcal{D}[\chi, \bar{\chi}] \mathcal{D}[\theta] \delta\left(\int_{x_{0}}^{x} d \xi_{\mu} \bar{\chi} \gamma^{\mu} \gamma_{5} \chi\right)\left|\operatorname{det}\left(-\frac{1}{\pi}\right)\right| e^{i \int d^{2} x \mathcal{L}_{1}^{\prime \prime}} \\
\mathcal{L}_{1}^{\prime \prime} & =\bar{\chi} i \not \partial \chi+\frac{1}{2 \pi} \partial_{\mu} \theta \partial^{\mu} \theta+V_{\mu} \frac{1}{\pi} \epsilon^{\mu \nu} \partial_{\nu} \theta-A_{\mu} \frac{1}{\pi} \partial^{\mu} \theta
\end{aligned}
$$


Note that effectively, inside the functional integral, the discussion above has shown that $\bar{\chi} \gamma_{\mu} \gamma_{5} \chi$ can be represented as the gradient of a field. It is due to this property, and to relation (6), that it is possible to eliminate two currents by a single constraint.

The fermion fields and the gauge fixing term have then totally decoupled from the $\theta$-integral which contains the vector and axial vector sources. Carrying out the $\chi$ integrations produces an unimportant constant factor $\mathcal{N}$, relating the normalization of the fermionic representation to the bosonic one.

If we rescale $\theta$ to get the usual canonical kinetic term and also change its sign, the generating functional becomes

$$
\begin{aligned}
\mathcal{Z}[V, A] & =\mathcal{N} \int \mathcal{D}[\theta] e^{i \int d^{2} x \mathcal{L}^{\prime \prime \prime}} \\
\mathcal{L}^{\prime \prime \prime} & =\frac{1}{2} \partial_{\mu} \theta \partial^{\mu} \theta-V_{\mu} \frac{1}{\sqrt{\pi}} \epsilon^{\mu \nu} \partial_{\nu} \theta+A_{\mu} \frac{1}{\sqrt{\pi}} \partial^{\mu} \theta
\end{aligned}
$$

Comparing the couplings to the external sources to those in (12), one finds the bosonization rules for the currents $(3)$ and $(7)$ with $\beta=2 \sqrt{\pi}$.

Although the whole discussion of path independence of the gauge fixing function $\Phi^{\prime}$ (once inside the functional integral) has pertained only to the case $\Delta=0$, it is obvious that the proof of path independence carries through for arbitrary $\Delta$.

\subsection{A smooth Cheshire Cat bag}

Until now, we have just recovered the well known bosonization rules for currents in $(1+1)$ dimensions. As we have seen, bosonization (and fermionization) occurs here not as a direct change of variables. Rather, we will in general always have both kinds of fields represented in the path integral, and only in certain very specific limits of the gauge fixing function do we achieve either a decoupling of fermions or zero boson fields. All interactions (couplings to the sources) now take place only through either bosonic or fermionic fields. The most interesting perspective in this connection is that we have not only shown equivalence between, e.g., the fermionic massless Thirring model and the free bosonic model, but that we have also shown how this equivalence extends to a whole continuum of theories involving interactively both bosons and fermions, corresponding to almost any arbitrary gauge fixing condition. All these theories are gauge equivalent.

However, our approach is flexible enough to also allow for a continuous change in representation from one region of space-time to another, choosing different gauge fixing conditions in the different regions. Such a gauge is a realization of the Cheshire Cat principle in a general way: the form of the bag and its wall can be completely arbitrary; being defined by a gauge fixing condition it is by construction an unphysical and unobservable object. For the purpose of achieving this, we simply choose $\Delta$ to be a function in space-time, interpolating between the limits 0 and 1 , which were discussed above. The Faddeev-Popov determinant remains unchanged; however, if $\Delta$ is not a constant, we have to be careful about the implications on the axial current. For example, the gauge (26) yields

$$
\Delta(x) j_{5}^{\mu}(x)+(1-\Delta(x)) \frac{1}{\pi} \partial^{\mu} \theta(x)+\left(\int_{x_{0}}^{x} d \xi_{\nu} j_{5}^{\nu}(\xi)-\frac{1}{\pi} \theta(x)\right) \partial^{\mu} \Delta(x)=0,
$$

where we abbreviate the fermionic currents from now on as

$$
j_{5}^{\mu}(x)=\bar{\chi}(x) \gamma^{\mu} \gamma_{5} \chi(x) \quad, \quad j^{\mu}(x)=\bar{\chi}(x) \gamma^{\mu} \chi(x)
$$


Eq.(39) already indicates that due to our gauge choice the components of the axial currents normal ${ }^{3)}$ to the bag surface need not be continuous.

Let us now illustrate the implications of the gauge (26) by examining some Ward identities. In particular, we look at a shift in $\theta(x)$. From the representation (16) of the generating functional $\mathcal{Z}[V, A]$, we derive

$$
\left\langle\partial_{\mu} j_{5}^{\mu}(x)-\frac{1}{\pi} \partial^{2} \theta(x)+\frac{1}{\pi}(1-\Delta(x)) b(x)\right\rangle+\frac{1}{\pi} \partial_{\mu}\left(\epsilon^{\mu \nu} V_{\nu}(x)+A^{\mu}(x)\right)=0,
$$

where the field $b(x)$ is the Lagrange multiplier field related to the gauge $\Phi^{\prime}$; the symbols $\langle\ldots\rangle$ denote the expectation value as calculated by functional integration. Similarly, we get from a chiral rotation of $\chi(x)$ and $\bar{\chi}(x)$ :

$$
\left\langle\partial_{\mu} j_{5}^{\mu}(x)-\frac{1}{\pi} \partial^{2} \theta(x)-\frac{1}{\pi} \Delta(x) b(x)\right\rangle+\frac{1}{\pi} \partial_{\mu}\left(\epsilon^{\mu \nu} V_{\nu}(x)+A^{\mu}(x)\right)=0 .
$$

In addition, we have the gauge constraint $\Phi^{\prime}$. It yields the following identity:

$$
\begin{gathered}
\left\langle\Delta(x) \partial_{\mu} j_{5}^{\mu}(x)+2 j_{5}^{\mu}(x) \partial_{\mu} \Delta(x)+\int_{x_{0}}^{x} d \xi_{\nu} j_{5}^{\nu}(\xi) \partial^{2} \Delta(x)\right. \\
\left.+(1-\Delta(x)) \frac{1}{\pi} \partial^{2} \theta(x)-2 \frac{1}{\pi} \partial^{\mu} \theta(x) \partial_{\mu} \Delta(x)-\frac{1}{\pi} \theta(x) \partial^{2} \Delta(x)\right\rangle=0 .
\end{gathered}
$$

Since another Ward identity reads

$$
\langle b(x)\rangle=0,
$$

eqs. (41) and (42) are in fact identical. The resulting identity and the gauge constraint (43) can now be written in an equivalent form:

$$
\begin{aligned}
\left\langle-\frac{1}{\pi} \partial^{2} \theta(x)-2\left(j_{5}^{\mu}(x)-\frac{1}{\pi} \partial^{\mu} \theta(x)\right) \partial_{\mu} \Delta(x)-\left(\int_{x_{0}}^{x} d \xi_{\nu} j_{5}^{\nu}(\xi)-\frac{1}{\pi} \theta(x)\right) \partial^{2} \Delta(x)\right\rangle & \\
+\Delta(x) \frac{1}{\pi} \partial_{\mu}\left(\epsilon^{\mu \nu} V_{\nu}(x)+A^{\mu}(x)\right) & =0, \\
\left\langle\partial_{\mu} j_{5}^{\mu}(x)+2\left(j_{5}^{\mu}(x)-\frac{1}{\pi} \partial^{\mu} \theta(x)\right) \partial_{\mu} \Delta(x)+\left(\int_{x_{0}}^{x} d \xi_{\nu} j_{5}^{\nu}(\xi)-\frac{1}{\pi} \theta(x)\right) \partial^{2} \Delta(x)\right\rangle & \\
+(1-\Delta(x)) \frac{1}{\pi} \partial_{\mu}\left(\epsilon^{\mu \nu} V_{\nu}(x)+A^{\mu}(x)\right) & =0 .
\end{aligned}
$$

If one takes the sum of both equations one recovers (41) and (42), respectively; if one takes the first equation multiplied with $(1-\Delta(x))$ and subtracts it from the second equation multiplied by $\Delta(x)$, one recovers $(43)$.

First of all, one observes that the fields $\theta(x)$ and $\chi(x)$ interact with the external sources in complementary regions defined by $\Delta(x)$ being 1 or 0 , and partly for all values in between. This is exactly how the gauge was originally choosen; the value of $\Delta$ gives that fraction of the physical axial current which should be represented by $\theta(x)$. In addition, there are terms in the equation of motion for $\theta(x)$ and in the equation for the divergence of $j_{5}^{\mu}(x)$ which are restricted to the transition region where $\Delta(x)$ varies from 0 to 1 . These terms appear with an opposite sign in both equations, and thus cancel when constructing the physical quantity $\partial_{\mu} J_{5}^{\mu}=\partial_{\mu} j_{5}^{\mu}-\frac{1}{\pi} \partial^{2} \theta$.

3) The term $\partial^{\mu} \Delta(x)$ defines the normal vector to the (in general, soft) bag surface 
What is the meaning of these terms? Let us define a quantity $\Sigma(x)$ as

$$
\Sigma(x)=\left\langle\int_{x_{0}}^{x} d \xi_{\nu} j_{5}^{\nu}(\xi)-\frac{1}{\pi} \theta(x)\right\rangle .
$$

This quantity is related to the physical axial current $J_{5}^{\mu}$ by

$$
\partial^{\mu} \Sigma(x)=\left\langle J_{5}^{\mu}(x)\right\rangle=\left\langle j_{5}^{\mu}(x)-\frac{1}{\pi} \partial^{\mu} \theta(x)\right\rangle .
$$

As such, it is independent of $\Delta(x)$. The equations (41) and (42) then provide the equation of motion for $\Sigma(x)$ :

$$
\partial^{2} \Sigma(x)+\frac{1}{\pi} \partial_{\mu}\left(\epsilon^{\mu \nu} V_{\nu}(x)+A^{\mu}(x)\right)=0,
$$

where again we have taken into account eq.(44). The terms in (45) depending on derivatives of $\Delta(x)$ arise because of the fact that, due to our gauge fixing function $\Phi^{\prime}$,

$$
\langle\theta(x)\rangle=-\pi \Delta(x) \Sigma(x) \quad, \quad\left\langle\int_{x_{0}}^{x} d \xi_{\nu} j_{5}^{\nu}(\xi)\right\rangle=(1-\Delta(x)) \Sigma(x) .
$$

Thus, $\Delta(x)$ switches on the field $\theta(x)$ as it changes from 0 to 1 ; at the same time, it constrains the fermion fields. The corresponding currents can be obtained as the gradients of the terms in (49), and therefore contain additional terms from the gradient acting on $\Delta(x)$.

Let us now consider the particular case of a Cheshire Cat bag as described in [4]. This corresponds to the choice

$$
\Delta(x)=\Theta(\mathbf{x}-\mathbf{z}),
$$

where $\Theta(y)$ is a step function and $\mathrm{x}$ denotes the spatial coordinate. The bag wall is then located at $\mathbf{z}$. The gauge fixing term $\Phi^{\prime}[\theta, \ldots]$ in $(26)$ therefore covers three domains: For $\mathbf{x}>\mathbf{z}$, we have $\Delta(x)=1$ and only the bosons couple to the external sources. For $\mathbf{x}<\mathbf{z}$, we have $\Delta(x)=0$ and only the fermions couple to the external sources. The total currents coupling to $A_{\mu}(x)$ and $V_{\mu}(x)$ are of course gauge invariant quantities and therefore independent of $\Delta(x)$. The additional surface terms in (39) thus indicate a possible mismatch of the fermionic and the bosonic current exactly on the bag surface. In particular, the normal ${ }^{4)}$ component of the axial currents and correspondingly the tangential component of the vector currents can be mismatched. In general, these terms causing the mismatch do not vanish over the whole range of the bag surface, but instead provide the interpolating currents between the two different regimes. The Cheshire Cat criterion, stating undisturbed flow of physical information across the bag surface, is always fullfilled by construction; the bag is just a gauge artefact. ${ }^{5)}$

4) For this case $\partial_{\mu} \Delta(x)=n_{\mu} \delta(\mathbf{x}-\mathbf{z})$ where $n_{\mu}$ is the vector normal to the bag surface pointing into the region with $\Delta(x)=1$.

5) This particular Cheshire Cat bag model involves a bag of interacting fermions (with no scalar field), described on the outside by a bosonic field and a decoupled fermionic field (which is even highly constrained by the gauge fixing function). This is not completely analogous to usual bag models, where one would wish to integrate out the fermions in the region in which they play no role. Such a procedure would induce boundary conditions on the bag surface. It would be interesting to check if in some special cases one recovers the usual hybrid bag model boundary conditions, as is implied by the analysis of ref. [4]. 
For the case of a sharp transition region, we can, however, look at the currents infinitesimally close to the bag surface. In this limit, we still have constant $\Delta(x)$ equal to 0 on the side respectively 1 on the other side. Eq.(49) therefore implies that the fermionic current equals the bosonic current in this specific limit. Only on the bag surface itself do we have additional singular currents from the rapid change of $\Delta(x)$. These have the opposite sign for $-\frac{1}{\tau} \partial^{\mu} \theta(x)$ and $j_{5}^{\mu}(x)$ as can easily be derived from (49); for the physical axial current given by their sum these singular surface currents thus cancel.

\section{Low and High Energy Fields}

\subsection{A NONLOCAL GAUGE}

Let us now consider another gauge fixing function, one which is suited for a distinction between low and high energy effective fields. Although we are working in $(1+1)$ dimensions, we shall again have a more physical setting in mind. We will therefore concentrate on a formulation in which fermionic ("quark") degrees of freedom are manifest at high energy, while bosonic ("meson") degrees of freedom appear dominant in the long wavelength limit. In $(1+1)$ dimensions, where we know (and have seen here in a different way) that we have complete bosonization, such a distinction is bound to be merely a question of language. So whereas no dynamics favors one formulation over the other, we nevertheless here have the complete freedom to choose convenient representations of high and low energy variables. This can give us a kind of smooth bag in momentum space, with many similarities to the believed behavior of quark degrees of freedom at high and low energy in the real world of strong interactions.

We implement this "momentum space bag" by choosing the divergence of the current $-\frac{1}{\pi} \partial^{\mu} \theta$ to be a fraction of the divergence of the physical current $J_{5}^{\mu}$ in momentum space, corresponding to a nonlocal relation:

$$
\begin{aligned}
\Phi^{n l}[\theta, \bar{\chi}, \chi] & =\partial_{\mu}\left(\frac{1}{\pi} \partial^{\mu} \theta+\int d^{2} y \Delta(x-y) J_{5}^{\mu}(y)\right) \\
& =\partial_{\mu} \int d^{2} y\left(\Delta(x-y) \bar{\chi}(y) \gamma^{\mu} \gamma_{5} \chi(y)+(\delta(x-y)-\Delta(x-y)) \frac{1}{\pi} \partial^{\mu} \theta(y)\right) \\
& =0 .
\end{aligned}
$$

In the last line, we have again used the definition of $J_{5}^{\mu}$ in (19). As in the last section $\Phi^{n l}[\ldots]$ depends not only on $\theta$, but also on the fermion fields.

This gauge has two main advantages: It respects translational invariance and as in the last section, the Faddeev-Popov determinant is independent of $\Delta(x-y)$. The calculation of this determinant proceeds in exactly the same way as for (21). We get

$$
\operatorname{det}\left(\frac{\delta \Phi^{n l}}{\delta \alpha}\right)=\operatorname{det}_{(0)}\left(-\frac{1}{\pi} \partial^{2}\right) \quad
$$

where the index $(0)$ again indicates that we restrict ourselves to non-zero modes. In addition, we have to gauge fix the zero modes.

For one possible interpretation of this gauge, assume that $\Delta(x-y)$ vanishes at distances larger than some scale $1 / M$. It therefore provides a smooth cutoff of 2 -momentum at $p^{2}=M^{2}$. We will demonstrate how this may lead to a description of the short distance physics by fermions, with the bosons being responsible for the long distance dynamics. Clearly, such a separation is ad hoc, with no real motivation from our previous discussion. In particular, since both the boson and fermion descriptions are really just different gauge 
choices, we should not be able to extract physical significance out of such a distinction between high and low energy fields. Nevertheless, we feel that it may be useful to have an example where a differentiation into high energy quark and low energy meson degrees of freedom can be made in such an explicit manner. Furthermore, we should also have in mind that one usually chooses a gauge to simplify the calculations or the physical picture. This particular gauge provides us with a quasi-particle picture (almost free fermions at high energies, semiclassical approximation for bosons at low energies) which is suggested by e.g. the so-called "cloudy bag" models.

In order to illustrate this idea in a more concrete manner, consider the following choice for $\Delta(p)$

$$
\Delta(p)=\left(1+\left(\frac{p^{2}}{M^{2}}\right)^{2}\right)^{-1}
$$

which interpolates smoothly between the limits $\Delta(0)=1$ and $\Delta(p) \lim _{p^{2} \rightarrow \infty} 0$. Here, we used a Fourier transformed representation which simplifies also the nonlocal gauge fixing term:

$$
\Phi_{p}^{n l}[\theta, \bar{\chi}, \chi]=\Delta(p) i p_{\mu} j_{5}^{\mu}(p)+(1-\Delta(p)) \frac{1}{\pi} p^{2} \theta(p),
$$

where $j_{5}^{\mu}(p)$ is the Fourier transform of the fermionic axial current,

$$
j_{5}^{\mu}(p)=\int d^{2} x e^{i p_{\nu} x^{\nu}} \bar{\chi}(x) \gamma^{\mu} \gamma_{5} \chi(x) .
$$

In the momentum representation, we also get a clue as to how this gauge works. For small $p^{2}$, the function $\Delta(p)$ tends to 1 , thus yielding the constraint $p_{\mu} j_{5}^{\mu}(p) \rightarrow 0$. If $p^{2}$ becomes large and therefore $\Delta(p)$ equal to zero, we get the condition $p^{2} \theta(p)=0$. This excludes modes $\theta(p)$ which create large gradients: the boson field is smooth. On the other hand, components of the fermionic axial current transferring low 2-momenta have to vanish. This means that the excitations of fermions into states differing by a small 2 -momentum $p_{\mu}$ are suppressed through the gauge; these processes are described by the boson field $\theta(p)$.

The usefulness of this particular gauge becomes obvious when we again look at some Ward identities, namely those corresponding to variations of $\theta, b$ and chiral rotations of the $\chi$ and $\bar{\chi}$. The auxiliary field $b$ is introduced just as in section 3 . We then get for the expectation values of the Fourier transforms:

$$
\begin{aligned}
\left\langle p_{\mu}\left(j_{5}^{\mu}(p)+\frac{i}{\pi} p^{\mu} \theta(p)\right)+\frac{1}{\pi} p_{\mu}\left(A^{\mu}(p)+\epsilon^{\mu \nu} V_{\nu}(p)\right)+(1-\Delta(p)) p^{2} b(p)\right\rangle & =0, \\
\left\langle\Delta(p) p_{\mu} j_{5}^{\mu}(p)+\frac{1}{\pi}(1-\Delta(p)) p^{2} \theta(p)\right\rangle & =0, \\
\left\langle p_{\mu}\left(j_{5}^{\mu}(p)+\frac{i}{\pi} p^{\mu} \theta(p)\right)+\frac{1}{\pi} p_{\mu}\left(A^{\mu}(p)+\epsilon^{\mu \nu} V_{\nu}(p)\right)-\Delta(p) p^{2} b(p)\right\rangle & =0 .
\end{aligned}
$$

Again, we can use the other Ward identity

$$
\langle b(p)\rangle=0,
$$

with the help of which the first and the last Ward identity in (56) are identical. The residual set of equations can be rewritten as

$$
p_{\mu}\left\langle j_{5}^{\mu}(p)\right\rangle=(1-\Delta(p)) \frac{1}{\pi} p_{\mu}\left(A^{\mu}(p)+\epsilon^{\mu \nu} V_{\nu}(p)\right),
$$




$$
\frac{1}{\pi} p^{2}\langle\theta(p)\rangle=-i \Delta(p) \frac{1}{\pi} p_{\mu}\left(A^{\mu}(p)+\epsilon^{\mu \nu} V_{\nu}(p)\right)
$$

This justifies the interpretation given above: The fermions see the sources as dressed by a form factor $(1-\Delta(p))$ and thus feel them only for large $p^{2}$. For the bosons, the sources appear with the complementary form factor $\Delta(p)$, and are only visible for small $p^{2}$. Due to the nonlocal structure of the gauge (51), this time we get no contributions from derivatives acting on $\Delta(x-y)$.

\subsection{The massless Schwinger model}

Let us illustrate this approach with the example of the massless Schwinger model which can be regarded as massless QED in $(1+1)$ dimensions. The Lagrangian is given by

$$
\mathcal{L}_{S}=\bar{\psi}(i \not \partial+V+e \not A) \psi-\frac{1}{4} F_{\mu \nu} F^{\mu \nu}
$$

In addition to the coupling to the "photon" field $A_{\mu}$, the fermions are also coupled to an external vector source $V_{\mu}$. The charge $e$ has dimension of mass; the field strength tensor is defined as usual, $F_{\mu \nu}=\partial_{\mu} A_{\nu}-\partial_{\nu} A_{\mu}$. Consider Landau gauge $\partial_{\mu} A^{\mu}=0$.

Next, we introduce the field $\theta$ as described above. The corresponding Lagrangian now reads

$$
\begin{aligned}
\mathcal{L}_{S}^{\prime}= & \bar{\chi}\left(i \not \partial+V+e \not A-\not \partial \theta \gamma_{5}\right) \chi-\frac{1}{4} F_{\mu \nu} F^{\mu \nu} \\
& +\frac{1}{2 \pi} \partial_{\mu} \theta \partial^{\mu} \theta+V_{\mu} \frac{1}{\pi} \epsilon^{\mu \nu} \partial_{\nu} \theta+e A_{\mu} \frac{1}{\pi} \epsilon^{\mu \nu} \partial_{\nu} \theta .
\end{aligned}
$$

Let us now look at some Ward identities. In principle, we still have the equations $(56)$, except that we have to replace the combination $A^{\mu}+\epsilon^{\mu \nu} V_{\nu}$ by $\epsilon^{\mu \nu}\left(V_{\nu}+e A_{\nu}\right)$.

A Ward identity derived as before gives:

$$
\left\langle p_{\mu}\left(j_{5}^{\mu}(p)+\frac{i}{\pi} p^{\mu} \theta(p)\right)+\frac{1}{\pi} p_{\mu} \epsilon^{\mu \nu}\left(e A_{\nu}(p)+V_{\nu}(p)\right)\right\rangle=0
$$

together with the gauge condition (54). In addition we get identities from a variation of $A_{\mu}$ and from its gauge condition:

$$
\begin{aligned}
-\left(p^{2} g^{\mu \nu}-p^{\mu} p^{\nu}\right)\left\langle A_{\nu}(p)\right\rangle+i p^{\mu}\left\langle b_{L}(p)\right\rangle+e\left\langle j^{\mu}(p)\right\rangle-i \frac{e}{\pi} \epsilon^{\mu \nu} p_{\nu}\langle\theta(p)\rangle & =0 \\
p_{\mu}\left\langle A^{\mu}(p)\right\rangle & =0 .
\end{aligned}
$$

Here $b_{L}(p)$ is the Lagrange multiplier field of the Lorentz gauge for $A_{\mu}(p) ; j^{\mu}(p)$ is the Fourier transform of the fermionic vector current $\bar{\chi} \gamma^{\mu} \chi$. From these two equations we easily derive

$$
p^{2} \epsilon^{\mu \nu} p_{\nu}\left\langle A_{\nu}(p)\right\rangle-i e p_{\mu}\left\langle j_{5}^{\mu}(p)\right\rangle+\frac{e}{\pi} p^{2}\langle\theta(p)\rangle=0 .
$$

This relation can be combined with the gauge constraint (54) leading to the following equations:

$$
\begin{aligned}
-e p_{\mu}\left\langle j_{5}^{\mu}(p)\right\rangle & =(1-\Delta(p)) p^{2} \epsilon^{\mu \nu} p_{\mu}\left\langle A_{\nu}(p)\right\rangle, \\
\frac{e}{\pi}\langle\theta(p)\rangle & =i \Delta(p) \epsilon^{\mu \nu} p_{\mu}\left\langle A_{\nu}(p)\right\rangle .
\end{aligned}
$$


In a similar manner, eq. (61) can be combined with the gauge condition yielding:

$$
\begin{aligned}
p_{\mu}\left\langle j_{5}^{\mu}(p)\right\rangle & =(1-\Delta(p)) \frac{1}{\pi} p_{\mu} \epsilon^{\mu \nu}\left(V_{\nu}(p)+e\left\langle A_{\nu}(p)\right\rangle\right), \\
\frac{1}{\pi} p^{2}\langle\theta(p)\rangle & =-i \Delta(p) \frac{1}{\pi} p_{\mu} \epsilon^{\mu \nu}\left(V_{\nu}(p)+e\left\langle A_{\nu}(p)\right\rangle\right) .
\end{aligned}
$$

Up to constant factors, the second equation of (64) identifies $\theta(p)$ with the low momentum components of the electric field. We can use this equation to rewrite the second equation of $(65)$ as

$$
\frac{1}{\pi}\left(p^{2}-\frac{e^{2}}{\pi}\right)\langle\theta(p)\rangle=-i \Delta(p) \frac{1}{\pi} p_{\mu} \epsilon^{\mu \nu} V_{\nu}(p)
$$

This equation identifies $\theta(p)$ as an effective low energy field with mass $e / \sqrt{\pi}$. It is coupled to the external source $V_{\mu}(p)$ only for momenta $p^{2}$ smaller than some scale $M^{2}$. This scale is of course unphysical; we could, however, completely bosonize the model (i.e., take $M^{2} \rightarrow \infty$ ) resulting in a free massive pseudoscalar field. This is also the exact bosonized version of the massless Schwinger model.

\section{The Massive Case}

The massless Thirring model is equivalent to a free bosonic field, and in that sense may seem too simple to test our ideas. It is, therefore, worthwhile to see to what extent we can support the notion of smooth bosonization beyond this particular model. A highly non-trivial extension consists in simply adding scalar and pseudoscalar mass terms to the Thirring model, which we now consider.

It is convenient to keep the discussion more general by introducing local sources $M_{S}(x)$ and $M_{P}(x)$ which couple to the scalar and pseudoscalar fermion densities $\bar{\psi} \psi$ and $\bar{\psi} \gamma_{5} \psi$, respectively. So, we add a term

$$
\mathcal{L}_{\text {mass }}=M_{S}(x) \bar{\psi}(x) \psi(x)+i M_{P}(x) \bar{\psi}(x) \gamma_{5} \psi(x)
$$

to the generating functional of eq. (12).

After the chiral rotation (13), we find that classically the Lagrangian changes as

$$
\mathcal{L}_{\text {mass }} \rightarrow M_{+} e^{2 i \theta} \bar{\chi} P_{+} \chi+M_{-} e^{-2 i \theta} \bar{\chi} P_{-} \chi,
$$

where

$$
M_{ \pm}(x) \equiv M_{S}(x) \pm i M_{P}(x),
$$

and where $P_{ \pm} \equiv \frac{1}{2}\left(1 \pm \gamma_{5}\right)$ are the usual chiral projectors.

In addition, we have to take into account the new change in the fermionic measure. As in the previous simpler case, this Jacobian is regularization scheme dependent, but we shall again adhere to a scheme in which the vector current is conserved. This Jacobian $J$ is then uniquely given $\mathrm{by}^{6}$ )

$$
\begin{aligned}
\ln (J)= & M_{S}\left\{\frac{\kappa_{1}(\Lambda)}{2 \pi}(\cos (2 \theta)-1)\right\}-M_{P}\left\{\frac{\kappa_{1}(\Lambda)}{2 \pi} \sin (2 \theta)\right\} \\
& -\frac{1}{2 \pi}\left\{M_{S} \cos (2 \theta)-M_{P} \sin (2 \theta)\right\}^{2}+\frac{1}{2 \pi} M_{S}^{2} \\
= & M_{+} \frac{\kappa_{1}(\Lambda)}{4 \pi}\left(e^{2 i \theta}-1\right)+M_{-} \frac{\kappa_{1}(\Lambda)}{4 \pi}\left(e^{-2 i \theta}-1\right) \\
& -\frac{1}{8 \pi} M_{+}^{2}\left(e^{4 i \theta}-1\right)-\frac{1}{8 \pi} M_{-}^{2}\left(e^{-4 i \theta}-1\right) .
\end{aligned}
$$

6) These terms can be extracted from the general expressions given in ref. [10]. 
This results from a Pauli-Villars regularization scheme. The two regulators which are needed have masses $k_{i} \Lambda$ and are weighted with factors $c_{i}$ fulfilling

$$
c_{1} k_{1}+c_{2} k_{2}=0 \quad, \quad c_{1}+c_{2}=1
$$

The cut-off dependence is then contained in the quantities

$$
\kappa_{n}(\Lambda)=\Lambda^{n} \sum_{i} c_{i} k_{i}^{n} \log \frac{k_{i}^{2} \Lambda^{2}}{\mu^{2}},
$$

where $\mu$ is a scale introduced by the regularization procedure. If we use the values of ref.[11], $c_{1}=c_{2}=\frac{1}{2}$ and $k_{1}=-k_{2}=1$ we get $\kappa_{1}(\Lambda)=0$.

The complete change in the Lagrangian due to the term (68) is then

$$
\begin{aligned}
\Delta \mathcal{L}_{\text {mass }}= & 2 M_{+}\left(e^{2 i \theta}-1\right) \bar{\chi} P_{+} \chi+2 M_{-}\left(e^{-2 i \theta}-1\right) \bar{\chi} P_{-} \chi \\
& +M_{+} \frac{\kappa_{1}(\Lambda)}{4 \pi}\left(e^{2 i \theta}-1\right)+M_{-} \frac{\kappa_{1}(\Lambda)}{4 \pi}\left(e^{-2 i \theta}-1\right) \\
& -\frac{1}{8 \pi} M_{+}^{2}\left(e^{4 i \theta}-1\right)-\frac{1}{8 \pi} M_{-}^{2}\left(e^{-4 i \theta}-1\right) .
\end{aligned}
$$

Note that, in these variables, there is complete decoupling between the $M_{+}$and $M_{-}$terms.

If we set $\Delta=0$, i.e., $\theta(x)=0$, we find $\Delta \mathcal{L}_{\text {mass }}=0$, and we simply recover the original fermionic theory, as we should. If we set $\Delta=1$, the fermionic currents $\bar{\chi} \gamma^{\mu} \chi$ and $\bar{\chi} \gamma^{\mu} \gamma^{5} \chi$ can be removed, just as explained in the section 3. But, in addition to the kinetic term of the fermions, we are obviously still left with the non-trivial fermion-boson interaction represented by

$$
\mathcal{L}_{\text {int }}=M_{+} e^{2 i \theta} \bar{\chi} P_{+} \chi+M_{-} e^{-2 i \theta} \bar{\chi} P_{-} \chi,
$$

i.e, the fermions have not trivially decoupled, as in the massless case. It is, however, obvious that these couplings can produce the parameters of the corresponding bosonic theory, hopefully the Sine-Gordon model, once the fermions are integrated out.

Where are the missing bosonization relations for the mass terms? First of all, a counting of degrees of freedom should be made here. Originally, in the massless theory we recovered two bosonization relations, those corresponding to, say, eq. (7) (one relation for each value of the Lorentz index $\mu$ ). The corresponding relation for the vector current is obtained automatically due to the relation (6). This may appear surprising, since we only introduced one spurious degree of freedom (the field $\theta$ ), and we later only gauge fixed one degree of freedom. As we showed in section 3.1, the mechanism behind these bosonization relations is indeed rather subtle. We can never expect to have the bosonization relations for the currents fall out of the path integral as a $\delta$-function constraint involving bosons and fermions, since these bosonization relations partly assume the equation of motion. An important ingredient for this derivation was the conservation of the vector current and the assumption that the corresponding symmetry is not spontaneously broken. Then, only one gauge fixing constraint of the form $\Phi^{\prime}[\theta, \bar{\chi}, \chi]$ with $\Delta=1$ is strong enough to effectively set the two fermionic currents to zero. When we finally look back at the remaining bosonic action, we read off the usual bosonization relations for these currents by comparing the coupling to the external sources. The same gauge fixing constraint $\Phi^{\prime}$ that just removes the fermionic currents cannot, in the same straightforward manner, remove the interaction terms of eq. (74), in order to provide the missing bosonization relations of eq. (3). 
The results of Dorn [11] can presumably be used to show the consistency of the missing bosonization relations to all orders in a perturbative expansion in the mass terms. Demonstrating this consistency, and only within the perturbative framework, is clearly a more modest achievement. But, in fact, the whole equivalence between the Massive Thirring model and the Sine-Gordon model has to our knowledge never been established beyond this kind of perturbative expansion [1]. In any case, if one is in doubt as to the validity of these bosonization equivalences beyond perturbation theory, one can always use the transformed Lagrangian we have provided above. Although not in full bosonic form, this is also non-perturbatively equivalent to the massive Thirring model. In more general cases, where one is not interested in an exact bosonization of a fermionic theory, one can use approximations to calculate the parameters of the bosonic theory.

\section{$6 \quad$ Summary and Conclusion}

We have introduced a new approach to the derivation of effective field theories. This consists in introducing the effective fields as gauge degrees of freedom, and then gauge fixing (some of) the original fields. We are then in a position to choose the most useful representation out of the huge variety available by simply taking a convenient gauge. We tried to demonstrate the power of this method by rederiving the famous bosonization relations in $(1+1)$ dimensions, and by reproducing the more sophisticated case of the Cheshire Cat Bag. The corresponding Cheshire Cat criterion, requiring undisturbed flow of physical information across the bag surface, is fulfilled in this approach by construction; the bag is just introduced by a gauge fixing condition and therefore unphysical. The resulting bag model is defined continuously over all space-time, with just decoupling (or even zero) fields in some regions. There are therefore no extraneous boundary conditions on the surface separating the two regimes, and one cannot directly make contact to the known bag models which can be classified according to these boundary conditions. Only by integrating over the decoupled fields in part of space-time would one be able to recover boundary conditions on the bag surface.

The general method we have described here can be used to reinvestigate other aspects of bosonization in $(1+1)$ dimensions, such as the non-abelian case [12], which is known to have a path integral interpretation. But the technique is more general, and can also be applied to higher dimensional theories, where of course one in general cannot obtain complete decoupling of either fermions or bosons. Thus one can now construct exact Cheshire Cat bag models without knowing the exact bosonized version of the theory under consideration (if such a version exists at all). This holds in $(3+1)$ dimensions as well.

ACKNOWLEDGEMENT: The authors acknowledge helpful discussions with Andreas Wirzba and Danny Birmingham. The work of R. Sollacher was supported in part by Deutsche Forschungsgemeinschaft, grant no. So 256/1. 


\section{REFERENCES}

[1] S. Coleman, Phys. Rev. D11 (1975) 2088.

[2] A. Luther and I. Peschel, Phys. Rev.B9 (1974) 2911.

A. Luther and V. Emery, Phys. Rev. Lett.33 (1974) 589.

[3] S. Mandelstam, Phys. Rev.D11 (1975) 3026.

[4] S. Nadkarni, H.B. Nielsen and I. Zahed, Nucl. Phys.B253 (1985) 308.

S. Nadkarni and H.B. Nielsen, Nucl. Phys. B362 (1986) 1.

S. Nadkarni and I. Zahed, Nucl. Phys. B263 (1986) 23.

R.J. Perry and M. Rho, Phys. Rev. D34 (1986) 1169.

[5] J. Alfaro and P.H. Damgaard, Ann. Phys. (N.Y.)202 (1990) 398.

[6] A. Hosoya and K. Kikkawa, Nucl. Phys. B101 (1975) 271.

[7] K. Furuya, R.E. Gamboa Saravi and F.A. Schaposnik, Nucl. Phys. B208 (1982) 159. R.E. Gamboa Saravi, M.A. Muscietti, F.A. Schaposnik and J.E. Solomin, Ann. Phys. (N.Y) 157 (1984) 360.

C.M. Naon, Phys. Rev. D31 (1985) 2035.

L.C.L. Botelho, Phys. Rev. D33 (1986) 1195.

[8] A.V. Kulikov, Teor. Mat. Fiz. 54 (1983) 157.

[9] R. Sollacher and H. Hofmann, Z. Phys. A339 (1991) 1.

[10] R.D. Ball, Phys. Rep.182 (1989) 1.

[11] H. Dorn, Phys. Lett. B167 (1986) 86.

[12] E. Witten, Comm. Math. Phys. 92 (1984) 455.

P. DiVecchia, B. Durhuus and J.L. Petersen, Phys. Lett. B144(1984) 245.

Y. Frishman, Phys. Lett. B146 (1984) 204.

E. Abdalla and M.C.B. Abdalla, Nucl. Phys. B225 (1985) 392.

D. Gonzales and A.N. Redlich, Phys. Lett. B147 (1984) 150. 